\title{
Variational Form of Classical Portfolio Strategy and Expected Wealth for a Defined Contributory Pension Scheme
}

\author{
Charles I. Nkeki ${ }^{1}$, Chukwuma R. Nwozo ${ }^{2}$ \\ ${ }^{1}$ Department of Mathematics, University of Benin, Benin City, Nigeria \\ ${ }^{2}$ Department of Mathematics, University of Ibadan, Ibadan, Nigeria \\ Email: \{nkekicharles2003, crnwozo\}@yahoo.com
}

Received October 26, 2011; revised December 10, 2011; accepted December 18, 2011

\begin{abstract}
This paper examines the variational form of classical portfolio strategy and expected terminal wealth for a Pension Plan Member (PPM) in a Defined Contribution (DC) Pension scheme. The flows of contributions made by PPM are invested into a market that is characterized by a cash account and a stock. It was assumed that the growth rate of salary of PPM is a linear function of time. The present value of PPM's future contribution process was obtained. The optimal portfolio processes with inter-temporal hedging terms that offset any shocks to the stochastic cash inflows were established. The expected value of PPM's terminal wealth was obtained.
\end{abstract}

Keywords: Variational Form; Classical Portfolio Strategy; Expected Wealth; Defined Contribution; Pension Scheme; Pension Plan Member; Inter-Temporal Hedging Terms; Stochastic Salary

\section{Introduction}

This paper considers the variational form of classical portfolio strategy and expected terminal wealth for a DC pension scheme. It was assumed that the salary and risky asset are driven by a standard geometric Brownian motion. The growth rate of salary of PPM is assumed to be a linear function of time. A non-linear partial differential equation was derived and then, the optimal portfolio values for the PPM were determined using power utility function. In this paper, we focus on studying the optimal portfolio strategy based on a classical model: geometric Brownian motion model as in Equation (2). While in [1] and [2], they are using an optimal portfolio strategy based on the non-Gaussian models. They constructed optimal portfolios of variance swaps based on a variance Gamma correlated model. The portfolios of the variance swaps are optimized based on maximizing the distorted expectation given the index of acceptability.

In related literature, [3] examined the rationale, nature and financial consequences of two alternative approaches to portfolio regulations for the long term institutional investor sectors of life insurance and pension funds. [4] considered the deterministic life styling (the gradual switch from equities to bonds according to preset rules) which is a popular asset allocation strategy during the accumulation phase of a defined contribution pension plans which is designed to protect the pension funds from a catastrophic fall in the stock market just prior to retirement. They shown that this strategy, although easy to understand and implement can be highly suboptimal. [5] developed a model for analyzing the ex ante liquidity premium demanded by the holder of an illiquid annuity. The annuity is an insurance product that is similar to a pension savings account with both an accumulation and decumulation phase. They computed the yield needed to compensate for the utility welfare loss, which is induced by the inability to re-balance and maintain an optimal portfolio when holding an annuity. [6-8] considered the optimal design of the minimum guarantee in a defined contribution pension fund scheme. They studied the investment in the financial market by assuring that the pension fund optimizes its retribution which is a part of the surplus, which is the difference between the pension fund value and the guarantee. [9] studied optimal investment strategy for a defined contributory pension plan. They adopted dynamic optimization technique. [10] considered optimal portfolio strategies with minimum guarantee and inflation protection for a DC pension scheme. [11] studied the optimal portfolio and strategic lifecycle consumption process in a defined contributory pension plan.

The remainder of this paper is organized as follows. In Section 2, we present the financial market models, Section 3, presents the present value of the expected flow of 
future discounted premiums process. We also establish in this section, the dynamics of the values of wealth of the PPM. In Section 4, we present the valuation of PPM's wealth process. In Section 5, we present the optimization process and portfolio value of a PPM in pension scheme. Section 6, presents the expected wealth for a PPM up to terminal period. Section 7 presents a special case of optimal portfolio selection problem. Finally, Section 8 concludes the paper.

\section{Problem Formulation}

The Brownian motion $W(t)=\left(W_{\phi}(t), W_{S}(t)\right)^{\prime}$, $0 \leq t \leq T$ is a 2 -dimensional process, defined on a given probability space $\left(\Omega, F,\left\{F_{t}^{\phi}\right\}_{t>0},\left\{F_{t}^{S}\right\}_{t>0}, P\right)$, where $P$ is the real world probability measure and $\sigma_{S}$ and $\sigma_{I}$ are the volatility of stock and volatility of inflation with respect to changes in $W_{S}(t)$ and $W_{\phi}(t)$, respectively. $\mu$ is the appreciation rate for stock. Moreover, $\sigma_{S}$ and $\sigma_{I}$ are the volatilities for the stock and inflation respectively, referred to as the coefficients of the market and are progressively measurable with respect to the filtration $F$. We assume that the investor faces a market that is characterized by a risk-free asset (cash account) and risky asset, all of whom are tradeable. In this paper, we allow the stock price to be correlated to inflation. Also, we correlated the cash inflows to stock market risk. The dynamics of the underlying assets are given by (1) and (2)

$$
\begin{gathered}
\left\{\begin{array}{l}
\mathrm{d} B(t)=r B(t) \mathrm{d} t \\
B(0)=1
\end{array}\right. \\
\left\{\begin{array}{l}
\mathrm{d} S(t)=S(t)\left(\mu \mathrm{d} t+\sigma_{S} \mathrm{~d} W_{S}(t)+\sigma_{I} \mathrm{~d} W_{\varphi}(t)\right) \\
S(0)=s_{0}
\end{array}\right.
\end{gathered}
$$

where,

$r$ is the nominal interest rate,

$B(t)$ is the price of the riskless asset at time $t$,

$S(t)$ is the stock price process at time $t$,

Therefore, (2) can be rewritten as

$$
\left\{\begin{array}{l}
\mathrm{d} S(t)=S(t)(\mu \mathrm{d} t+\sigma \mathrm{d} W(t)), \\
S(0)=s_{0},
\end{array}\right.
$$

where, $\mathrm{d} W(t)=\left(\begin{array}{ll}\mathrm{d} W_{\varphi}(t) & \left.\mathrm{d} W_{S}(t)\right)^{\prime} \text { and }\end{array}\right.$ $\sigma=\left(\begin{array}{ll}\sigma_{I} & \sigma_{S}\end{array}\right) \neq 0$.

It is assume that the market is complete; therefore there exists a unique market price of risk $\theta$ satisfying

$$
\theta=\sigma^{-1}(\mu-r) \text {. }
$$

The salary of the PPM is assume to follow the dynamics

$$
\left\{\begin{array}{l}
\mathrm{d} \varphi(t)=\varphi(t)\left(\omega(t) \mathrm{d} t+\sigma_{\varphi} \mathrm{d} W(t)\right), \\
\varphi(0)=\varphi_{0}>0
\end{array}\right.
$$

where, $\sigma_{\varphi}=\left(\begin{array}{ll}\sigma_{1} & \sigma_{2}\end{array}\right), \sigma_{1}$ is the volatility caused by the source of inflation, $W_{\varphi}(t)$ and $\sigma_{2}$ is the volatility caused by the source of uncertainty arises from the stock market, $W_{S}(t)$. We assume that the growth rate of the salary of PPM is linear and satisfies

$\omega(t)=a+\beta t, a>0, \beta \geq 0 . \quad a \quad$ may be seen as expected yearly growth of salary and $\beta$ as effect of economic growth or welfare of the PPM.

Therefore, the flow of contributions of the PPM is given by $c \varphi(t), c>0$, where $c$ is proportion of PPM salary he/she is contributing into the scheme.

We now define the following exponential process which we assume to be Martingale in $P$ :

$$
Z(t)=\exp \left[-\theta^{\prime} W(t)-\frac{1}{2}\|\theta\|^{2} t\right]
$$

which will be useful in the next section.

Let $X(t)$ be the wealth process and $\Delta(t)$ the portfolio process, that is the proportion of wealth invested in stock at time $t$. Then, $\Delta_{0}(t)=1-\Delta(t)$ is the proportion wealth invested in the riskless asset at time $t$. We now have the following definition:

Definition 1: the portfolio process $\Delta$ is said to be selffinancing if the corresponding wealth process $X(t), t \in[0, T]$, satisfies

$$
\begin{aligned}
\mathrm{d} X(t)= & X(t)[(r+\Delta(t)(\mu-r)) \mathrm{d} t+\Delta(t) \sigma \mathrm{d} W(t)] \\
& +c \varphi(t) \mathrm{d} t .
\end{aligned}
$$

\section{The Expected Value of Future Contribution of a PPM}

Definition 2: the present value of expected future contribution process is defined as

$$
\Phi(t)=E_{t}\left[\int_{t}^{T} \frac{\Lambda(s)}{\Lambda(t)} c \varphi(s) \mathrm{d} s\right],
$$

where $E_{t}(\cdot)=E(. \mid F(t))$ is the conditional expectation with respect to the Brownian filtration $\{F(t)\}_{t \geq 0}$ and $\Lambda(t)=Z(t) \exp (-r t)$ is the stochastics discount factor which adjusts for nominal interest rate and market price of risks.

Theorem 1 tells us that the present value of expected future contribution process, then proportional to the instanttaneous contribution process $c \varphi($.).

Definition 3: a portfolio process is said to be admissible if the corresponding wealth process $X(t)$ satisfies 


$$
\begin{aligned}
& P\left(X(t)+E_{t}\left[\int_{t}^{T} \frac{\Lambda(s)}{\Lambda(t)} c \varphi(s) \mathrm{d} s\right] \geq 0\right)=1, \\
& \forall t \in[0, T] .
\end{aligned}
$$

Theorem 1: let $\Phi(t)$ be the present value of expected future contribution (PVFC) process, then

$$
\begin{aligned}
& \left\{\begin{array}{l}
\Phi(t)=\frac{c \varphi(t)}{\alpha}(\exp (\alpha(T-t))-1), \beta=0 \\
\Phi(0)=\frac{c \varphi_{0}}{\alpha}(\exp (\alpha T)-1), \beta=0
\end{array}\right. \\
\Phi(t)= & -\frac{c \varphi(t)}{2 \sqrt{\beta}}\left(\sqrt{\pi} \exp \left(-\frac{\alpha^{2}}{4 \beta}\right)\right. \\
& \left.\times\left(\operatorname{Erfi}\left[\frac{-2 \beta(T-t)-\alpha}{2 \sqrt{\beta}}\right]\right)+\operatorname{Erfi}\left[\frac{\alpha}{2 \sqrt{\beta}}\right]\right), \beta>0 \\
\Phi(0)= & -\frac{c \varphi_{0}}{2 \sqrt{\beta}}\left(\sqrt{\pi} \exp \left(-\frac{\alpha^{2}}{4 \beta}\right)\right. \\
& \left.\times\left(\operatorname{Erfi}\left[\frac{-2 \beta T-\alpha}{2 \sqrt{\beta}}\right]\right)+\operatorname{Erfi}\left[\frac{\alpha}{2 \sqrt{\beta}}\right]\right), \beta>0 \\
& (T)=0
\end{aligned}
$$

where, $\alpha=a-r-\left\|\sigma_{\phi}\right\|\|\theta\|$.
Proof: by definition,

$$
\begin{aligned}
\Phi(t) & :=E_{t}\left[\int_{t}^{T} \frac{\Lambda(s)}{\Lambda(t)} c \varphi(s) \mathrm{d} s\right], T \geq t \\
& =c \varphi(t) E_{t}\left[\int_{t}^{T} \frac{\Lambda(s)}{\Lambda(t)} \frac{\varphi(s)}{\varphi(t)} \mathrm{d} s\right] .
\end{aligned}
$$

The processes $\Lambda($.$) and \varphi($.$) are geometric Brow-$ nian motions. It therefore follows that $\varphi(.) \frac{\Lambda(s)}{\Lambda(t)} \frac{\varphi(s)}{\varphi(t)}$ is independent of $\{F(t)\}_{s \geq t}$. Hence,

$$
\begin{aligned}
\Phi(t)= & c \varphi(t) E\left[\int_{0}^{T-t} \frac{\Lambda(s)}{\Lambda(0)} \frac{\varphi(s)}{\varphi(0)} \mathrm{d} s\right] \\
= & c \varphi(t) E\left[\int_{0}^{T-t} \Lambda(s) \frac{\varphi(s)}{\varphi(0)} \mathrm{d} s\right] \\
= & c \varphi(t) E\left[\int_{0}^{T-t}(\exp (-r s) Z(s)\right. \\
& \left.\left.\times \exp \left(a s+\beta s^{2}-\frac{1}{2}\left\|\sigma_{\varphi}\right\|^{2} s+\sigma_{\varphi} W(s)\right) \mathrm{d} s\right)\right] \\
= & c \varphi(t)\left[\int_{0}^{T-t} \exp \left(\beta s^{2}+\alpha s\right) \mathrm{d} s\right]
\end{aligned}
$$

where, $\alpha=a-r-\left\|\sigma_{\varphi}\right\|\|\theta\|$.

Therefore,

$$
\begin{gathered}
\left\{\begin{array}{l}
\Phi(t)=\frac{c \varphi(t)}{\alpha}(\exp (\alpha(T-t))-1), \beta=0 \\
\Phi(0)=\frac{c \varphi_{0}}{\alpha}(\exp (\alpha T)-1), \beta=0
\end{array}\right. \\
\left\{\begin{array}{l}
\Phi(t)=-\frac{c \varphi(t)}{2 \sqrt{\beta}}\left(\sqrt{\pi} \exp \left(-\frac{\alpha^{2}}{4 \beta}\right) \times\left(\operatorname{Erfi}\left[\frac{-2 \beta(T-t)-\alpha}{2 \sqrt{\beta}}\right]\right)+\operatorname{Erfi}\left[\frac{\alpha}{2 \sqrt{\beta}}\right]\right), \beta>0 \\
\Phi(0)=-\frac{c \varphi_{0}}{2 \sqrt{\beta}}\left(\sqrt{\pi} \exp \left(-\frac{\alpha^{2}}{4 \beta}\right) \times\left(\operatorname{Erfi}\left[\frac{-2 \beta T-\alpha}{2 \sqrt{\beta}}\right]\right)+\operatorname{Erfi}\left[\frac{\alpha}{2 \sqrt{\beta}}\right]\right), \beta>0 \\
\text { case of } \beta=0, \text { see }[4,7,10-12] .
\end{array}\right. \\
\Phi(T)=0 .
\end{gathered}
$$

Lemma 1: the dynamics of the present value of future

Numerical Example 1: setting $\mu=0.1, \beta=0.0001$, $a=0.05, T=20, r=0.04, \varphi_{0}=50000, \sigma=0.25$, $\sigma_{\varphi}=0.23, \gamma=0.5, c=0.075$. Mathematica 6.0 was used for the plotting.

In this paper, we consider the case of $\beta>0$. For the contribution process is given by

$$
\mathrm{d} \Phi(t)=\Phi(t)\left(\kappa(t) \mathrm{d} t+\sigma_{\varphi} \mathrm{d} W(t)\right)-c \varphi(t) \mathrm{d} t
$$

where,

$$
A(t)=\sqrt{\pi}\left(\operatorname{Erfi}\left[\frac{-2 \beta(T-t)-\alpha}{2 \sqrt{\beta}}\right]+\operatorname{Erfi}\left[\frac{\alpha}{2 \sqrt{\beta}}\right]\right)
$$




$$
\kappa(t)=-\frac{\left((a+\beta t) A(t)+\exp \left(\frac{(-2 \beta(T-t)-\alpha)^{2}}{4 \beta}\right)-2 \sqrt{\beta} \exp \left(\frac{\alpha^{2}}{4 \beta}\right)\right)}{A(t)}, T>t .
$$

Proof: taking differential of both sides of (8), we obtain the following:

$$
\begin{aligned}
\mathrm{d} \Phi(t)=\mathrm{d}( & \left.-\frac{c \varphi(t)}{2 \sqrt{\beta}}\left(\sqrt{\pi} \exp \left(-\frac{\alpha^{2}}{4 \beta}\right) \times\left(\operatorname{Erfi}\left[\frac{-2 \beta(T-t)-\alpha}{2 \sqrt{\beta}}\right]\right)+\operatorname{Erfi}\left[\frac{\alpha}{2 \sqrt{\beta}}\right]\right)\right) \\
= & -\frac{c \sqrt{\pi} \varphi(t)}{2 \sqrt{\beta}} \exp \left(-\frac{\alpha^{2}}{4 \beta}\right) \times\left(\left(\operatorname{Erfi}\left[\frac{-2 \beta(T-t)-\alpha}{2 \sqrt{\beta}}\right]\right)+\operatorname{Erfi}\left[\frac{\alpha}{2 \sqrt{\beta}}\right]\right) \times\left(\omega(t) \mathrm{d} t+\sigma_{\varphi} \mathrm{d} W(t)\right) \\
& -\frac{c \varphi(t)}{2 \sqrt{\beta}}\left(\exp \left(\frac{(-2 \beta(T-t)-\alpha)^{2}}{4 \beta}-\frac{\alpha^{2}}{4 \beta}\right)\right) \\
= & -\frac{c \varphi(t)}{2 \sqrt{\beta}} \exp \left(-\frac{\alpha^{2}}{4 \beta}\right)\left\{A(t)\left(\omega(t) \mathrm{d} t+\sigma_{\varphi} \mathrm{d} W(t)\right)+\exp \left(\frac{(-2 \beta(T-t)-\alpha)^{2}}{4 \beta}\right) \mathrm{d} t\right\} \\
=- & \frac{c \varphi(t)}{2 \sqrt{\beta}} \exp \left(-\frac{\alpha^{2}}{4 \beta}\right)\left\{A(t)\left(\omega(t) \mathrm{d} t+\sigma_{\varphi} \mathrm{d} W(t)\right)+\exp \left(\frac{(-2 \beta(T-t)-\alpha)^{2}}{4 \beta}\right) \mathrm{d} t\right\}-c \varphi(t) \mathrm{d} t+c \varphi(t) \mathrm{d} t
\end{aligned}
$$

Therefore,

$$
\mathrm{d} \Phi(t)=\Phi(t)\left(\kappa(t) \mathrm{d} t+\sigma_{\varphi} \mathrm{d} W(t)\right)-c \varphi(t) \mathrm{d} t
$$

where,

$$
\kappa(t)=-\frac{\left(A(t)(a+\beta t)+\exp \left(\frac{(-2 \beta(T-t)-\alpha)^{2}}{4 \beta}\right)-2 \sqrt{\beta} \exp \left(\frac{\alpha^{2}}{4 \beta}\right)\right)}{A(t)}, T>t .
$$

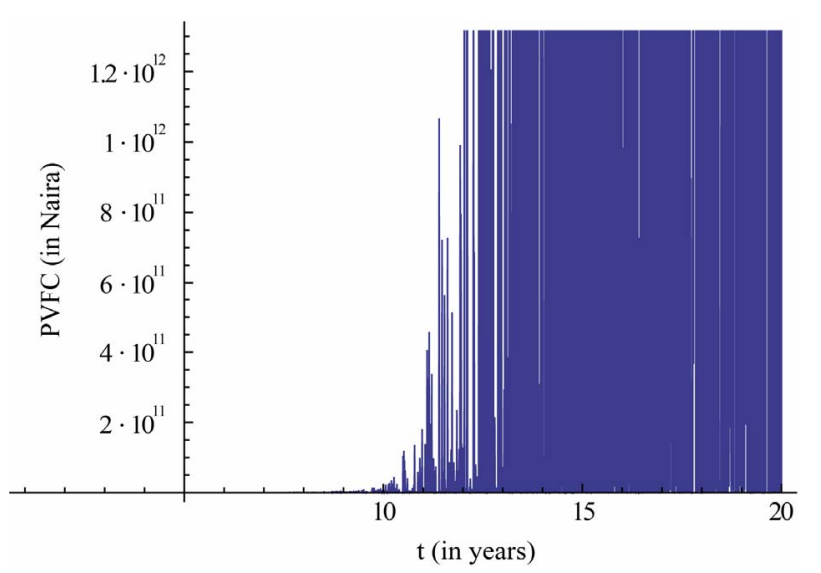

Figure 1. The present value of PPM's future contributions. The expected today value of PPM's contributions is obtained as 49,890 Naira. From the Figure 1, observed that the expected future contribution is highly volatile. This is because there is high rate of inflation. This, to a great extent will reduce the value of PPM's benefits at retirement.

\section{Valuation of PPM's Wealth Process}

Definition 2: the value of PPM's wealth $V(t)$ at time $t$ is the sum of PPM's wealth $X(t)$ and the present value of expected future contribution process, $\Phi(t)$ at time $t$ that is,

$$
V(t)=X(t)+\Phi(t), t \in[0, T] .
$$

Finding the differential of both sides of (11) and substituting (5) and (10), we obtain

$$
\begin{aligned}
\mathrm{d} V(t)= & {[X(t)(r+\Delta(t)(\mu-r))+\Phi(t) \kappa(t)] \mathrm{d} t } \\
& +\left(\Delta(t) X(t) \sigma+\sigma_{\phi} \Phi(t)\right) \mathrm{d} W(t)
\end{aligned}
$$

\section{The Optimization Process and Portfolio Value of a PPM}

In this section, we consider the optimal portfolio process for the IC. We define the general value function 


$$
J\left(t, V_{\Delta}, \Delta\right)=E\left[u\left(V_{\Delta}(T)\right) \mid X(t)=X, \Phi(t)=\Phi\right],
$$

where $V_{\Delta}(t)$ is the path of $V(t)$ given the portfolio strategy $\Delta$. Define $\Pi(V)$ to be the set of all admissible portfolio strategy that are $F_{V}$ - progressively measurable, and let $U(V(t))$ be a concave function in $V(t)$ such that

$$
\begin{aligned}
& U(V(t)) \\
& =\sup _{\Delta \in \Pi(V)} E\left[u\left(V_{\Delta}(T)\right) \mid X(t)=X, \Phi(t)=\Phi\right] \\
& =\sup _{\Delta \in \Pi(V)} J\left(t, V_{\Delta}, \Delta\right) .
\end{aligned}
$$

Then $U(V(t))$ satisfies the HJB equation

$$
U_{t}+\sup _{\Delta \in \Pi(V)} H(t, V, \Delta)=0
$$

subject to: $U(T, V)=\frac{V^{1-\gamma}}{1-\gamma}, \gamma \neq 1, \gamma>0$, where,

$$
\begin{aligned}
& H(t, V, \Delta)=(r X+(\mu-r) X \Delta(t)) U_{X} \\
& +\Phi \kappa(\tau) U_{\Phi}+\frac{1}{2} X^{2} \Delta(t)^{2} \sigma^{2} U_{X X} \\
& +X \Phi \Delta(t) \sigma \sigma_{\varphi} U_{X \Phi}+\frac{1}{2} \Phi^{2} \sigma_{\varphi}^{2} U_{\Phi \Phi} .
\end{aligned}
$$

Finding the partial derivative of $H(t, V, \Delta)$ with respect to the optimal control $\Delta(t)$ and setting it to zero, we obtain

$$
\Delta^{*}(t)=\frac{-(\mu-r) U_{X}-\Phi \sigma \sigma_{\varphi} U_{X \Phi}}{X \sigma^{2} U_{X X}} .
$$

Substituting (14) into (13), we obtain the HJB equation

$$
\begin{aligned}
& U_{t}+r X U_{X}-\frac{1}{2} \frac{(\mu-r)^{2} U_{X}^{2}}{\sigma^{2} U_{X X}}+\frac{\Phi^{2} \sigma_{\varphi}^{\prime 2} U_{X \Phi}^{2}}{U_{X X}} \\
& -\frac{\Phi \sigma_{\varphi}(\mu-r) U_{X} U_{X \Phi}}{\sigma U_{X X}}-\frac{1}{2} \Phi^{2} \sigma_{\varphi}^{\prime 2} U_{\Phi \Phi}+\Phi \kappa(\tau) U_{\Phi}=0 .
\end{aligned}
$$

Let $U(V(t))=\frac{(V g(t))^{1-\gamma}}{1-\gamma}, \gamma \neq 1, \gamma>0, \quad$ be the solution to the HJB Equation (15), then,

$$
\begin{gathered}
U_{t}(V(t))=V^{1-\gamma} g(t)^{-\gamma} g^{\prime}(t) \\
U_{X}(V(t))=V^{-\gamma} g(t)^{1-\gamma} \\
U_{X X}(V(t))=-\gamma V^{-\gamma-1} g(t)^{1-\gamma} \\
U_{X \Phi}(V(t))=-\gamma V^{-\gamma-1} g(t)^{1-\gamma}
\end{gathered}
$$

$$
\begin{gathered}
U_{\Phi}(V(t))=V^{-\gamma} g(t)^{1-\gamma} \\
U_{\Phi \Phi}(V(t))=-\gamma V^{-\gamma-1} g(t)^{1-\gamma}
\end{gathered}
$$

Substituting the partial derivatives above into (15), we obtain

$$
\begin{aligned}
& V g^{\prime}(t)+r X g(t)+\frac{1}{2} \frac{(\mu-r)^{2}}{\sigma^{2} \gamma} V g(t)+\Phi \kappa(\tau) g(t) \\
& -\frac{1}{2} \Phi^{2} \sigma_{\varphi}^{2} \gamma V^{-1} g(t)-\Phi \sigma_{\varphi}\left(\frac{\mu-r}{\sigma}\right) g(t)=0
\end{aligned}
$$

Again, substituting the partial derivatives into (14), we obtain

$$
\Delta^{*}(t)=\frac{(\mu-r)\left(X^{*}(t)+\Phi(t)\right)}{\gamma X^{*}(t) \sigma^{2}}-\frac{\Phi(t) \sigma_{\varphi}}{X^{*}(t) \sigma}
$$

Therefore, the portfolio value in the riskless asset is obtain as

$$
\Delta_{0}^{*}(t)=1+\frac{\Phi(t) \sigma_{\varphi}}{X(t) \sigma}-\frac{(\mu-r)\left(X^{*}(t)+\Phi(t)\right)}{\gamma X^{*}(t) \sigma^{2}} .
$$

Numerical Example 2:

Setting $\mu=0.1, \beta=0.0001, a=0.05, T=20$, $r=0.04, \varphi_{0}=50000, \sigma=0.25, \sigma_{\varphi}=0.23, \gamma=0.5$, $c=0.07$, we found that the optimal portfolio in stock at retirement to be 1.92 and in cash account to be -0.92 .

\section{Expected Wealth for a PPM}

In other to determine the expected wealth of the PPM at time $t$, we find the mathematical expectation of (12) as follows:

$$
\mathrm{d} E(V(t))=E(X(t)(r+\Delta(t)(\mu-r))+\Phi(t) \kappa(t)) \mathrm{d} t .
$$

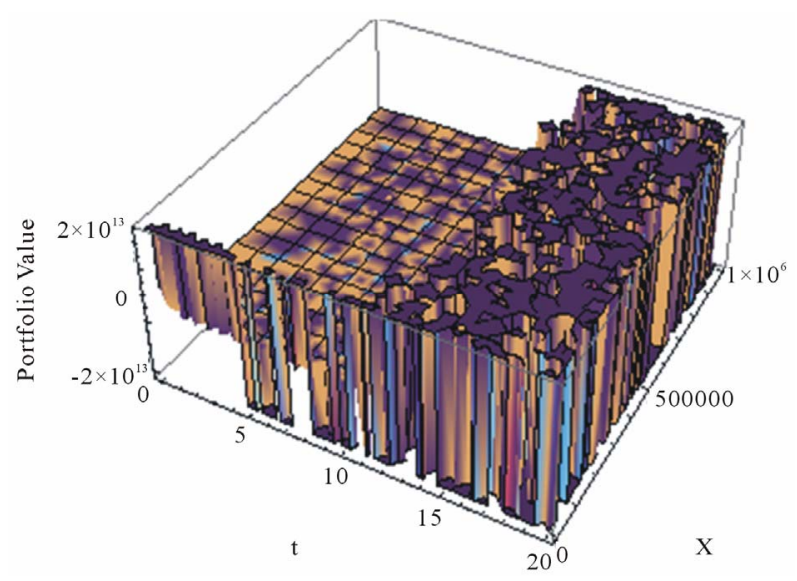

Figure 2. Portfolio value in stock. It has an optimal portfolio value after 20 years to be 1.92 . This implies that more funds should be borrowed from cash account and then, invests in stock market. 


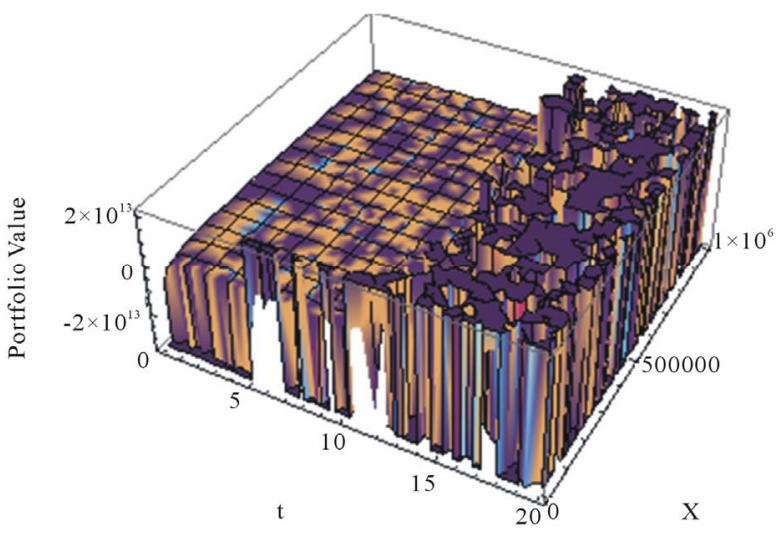

Figure 3. Portfolio value in cash account. It has an optimal portfolio value after 20 years to be $-\mathbf{0 . 9 2}$. This implies that no fund should keep in cash account at the optimal level and that all fund should remain in stock market.

For simplicity, we set $a=r+\delta, \delta \geq 0$, so that

$$
\begin{aligned}
& \mathrm{d} E(V(t))=\left(\left(r+\frac{(\mu-r)^{2}}{\gamma \sigma^{2}}\right) E(V(t))\right. \\
&\left.-\left(\frac{(\mu-r) \sigma_{\phi}}{\sigma}+\bar{\kappa}(t)\right) E(\Phi(t))\right) \mathrm{d} t \\
& \mathrm{~d} E(V(t))=\left(\left(r+\frac{(\mu-r)^{2}}{\gamma \sigma^{2}}\right) E(V(t))\right. \\
&-\left(\frac{(\mu-r) \sigma_{\varphi}}{\sigma}-\bar{\kappa}(t)\right) \frac{c \varphi_{0} \exp \left(\int(\omega(t)) \mathrm{d} t\right)}{2 \sqrt{\beta}} \\
&\left.\times\left(A(t) \exp \left(-\frac{\alpha^{2}}{4 \beta}\right)\right)\right) \mathrm{d} t \\
& \mathrm{~d} E(V(t))=\left(\left(r+\frac{\theta^{2}}{\gamma}\right) E(V(t))-G(t)\right) \mathrm{d} t
\end{aligned}
$$

where,

$$
\begin{aligned}
& G(t)=\left(\theta \sigma_{\phi}-\bar{\kappa}(t)\right) \frac{c \varphi_{0} \exp \left(a t+\frac{\beta t^{2}}{2}\right)}{2 \sqrt{\beta}} \\
& \times A(t) \exp \left(-\frac{\alpha^{2}}{4 \beta}\right), \\
& \bar{\kappa}(t)= \frac{((\delta+\beta t) A(t)+f(t))}{A(t)}, T>t, \\
& f(t)=\exp \left(\frac{(-2 \beta(T-t)-\alpha)^{2}}{4 \beta}\right)-2 \sqrt{\beta} \exp \left(\frac{\alpha^{2}}{4 \beta}\right) .
\end{aligned}
$$

Integrating both sides of (19), we obtain

$$
\begin{aligned}
& E(V(t))=\exp \left(\left(r+\frac{\sigma \theta^{2}}{\gamma}\right) t\right) \\
& \times\left(v_{0}-\frac{c \varphi_{0} \exp \left(\frac{-a^{2}}{4 \beta}\right)}{2 \sqrt{\beta}} \times \int_{0}^{t} A(s)\left(\sigma_{\varphi} \theta-\kappa(s)\right)\right. \\
& \left.\times \exp \left(\left(a-r-\frac{\theta^{2}}{\gamma}\right) s+\frac{\beta s^{2}}{2}\right) \mathrm{d} s\right) .
\end{aligned}
$$

At $t=T$, we have

$$
\begin{aligned}
& E(V(T))=\exp \left(\left(r+\frac{\sigma \theta^{2}}{\gamma}\right) T\right) \\
& \times\left(v_{0}-\frac{c \varphi_{0} \exp \left(\frac{-a^{2}}{4 \beta}\right)}{2 \sqrt{\beta}} \times \int_{0}^{T} H(s)\left(\sigma_{\varphi} \theta-\kappa(s)\right)\right. \\
& \left.\times \exp \left(\left(a-r-\frac{\theta^{2}}{\gamma}\right) s+\frac{\beta s^{2}}{2}\right) \mathrm{d} s\right) .
\end{aligned}
$$

Numerical Example 3: in this numerical example, we illustrate the expected value of a PPM's wealth up to retirement.

In Figure 4, we set $\varphi_{0}=50000, x_{0}=100, \delta=0.01$, $\mu=0.1, r=0.04, T=20, a=0.05, \sigma=0.25$, $\sigma_{\varphi} 0.23, \gamma=0.5, c=0.075$ and MATLAB was used for the plotting. It was found that at final time $T$, the

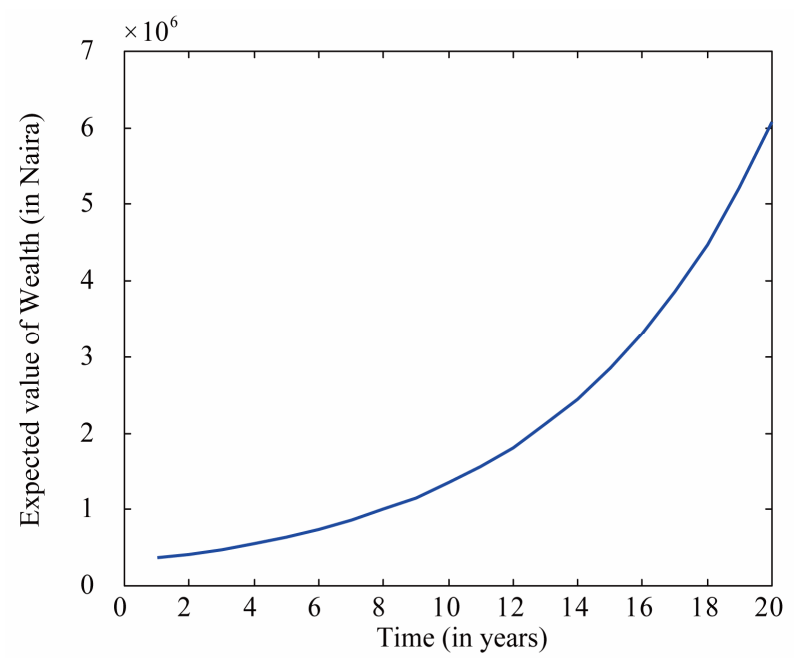

Figure 4. Expected value of wealth of a PPM. 
expected value of PPM's wealth is $6,078,720$ Naira.

\section{Discussion}

In Figure 1, we found that the expected present value of PPM's contributions is obtained as 49,890 Naira. We also observed that the expected future contribution is highly volatile. This is because there is high rate of inflation. In Figure 2, we found that the optimal portfolio value after 20 years to be 1.92. This implies that more funds should be borrowed from cash account and then, invests in stock market. In Figure 3, we found that the optimal portfolio value after 20 years to be -0.92 . This implies that no fund should be kept in cash account at the optimal level and that all fund should remain in stock market. Figure 4 shows the expected value of PPM's wealth up to 20 years and is obtained as $6,078,720$ Naira.

\section{Special Case $c=0$}

If we set $c=0$, we obtain the usual portfolio selection problem, provided that $x_{0}>0$. In that case, we have

$$
E(\tilde{V}(T))=E(X(T))=x_{0} \exp \left(\left(r+\frac{\sigma \theta^{2}}{\gamma}\right) T\right) .
$$

Corollary: assume that an investor intends to invest a wealth of $x_{0}>0$ for the time horizon $T>0$ in a financial market as in section (1) and wealth Equation (5). Assume that the investor maximizes the expected utility of final wealth at time $T$, then,

$$
E(X(T))=x_{0} \exp \left(\left(r+\frac{\sigma \theta^{2}}{\gamma}\right) T\right) .
$$

Observed that the ratio of the expected value of PPM's terminal wealth to the expected usual portfolio selection problem is obtain as

$$
\begin{aligned}
\frac{E(V(T))}{E(X(T))}= & \frac{v_{0}}{x_{0}}-\frac{c \varphi_{0} \exp \left(\frac{-a^{2}}{4 \beta}\right)}{2 x_{0} \sqrt{\beta}} \\
& \times \int_{0}^{T} H(s)\left(\sigma_{\phi} \theta-\kappa(s)\right) \\
& \times \exp \left(\left(a-r-\frac{\theta^{2}}{\gamma}\right) s+\frac{\beta s^{2}}{2}\right) \mathrm{d} s . \\
\frac{E(V(T))}{E(X(T))}-\frac{v_{0}}{x_{0}}+\frac{c \varphi_{0} \exp \left(\frac{-a^{2}}{4 \beta}\right)}{2 x_{0} \sqrt{\beta}} & \\
=\int_{0}^{T} H(s)\left(\sigma_{\varphi} \theta-\right. & \kappa(s)) \times \exp \left(\left(a-r-\frac{\theta^{2}}{\gamma}\right) s+\frac{\beta s^{2}}{2}\right) \mathrm{d} s .
\end{aligned}
$$

Substituting (23) into (21), we obtain

$$
E(V(T))=\frac{H(T)}{K(T)},
$$

where,

$$
\begin{aligned}
K(T) & =\frac{2 x_{0} \sqrt{\beta}+c \varphi_{0} \exp \left(\frac{-a^{2}}{4 \beta}\right)}{2 x_{0} \sqrt{\beta}}, \\
H(T)= & \exp \left(\left(r+\frac{\sigma \theta^{2}}{\gamma}\right) T\right) \\
& \times\left(v_{0}+\frac{c \varphi_{0} \exp \left(\frac{-a^{2}}{4 \beta}\right)}{2 x_{0} \sqrt{\beta}}\left(v_{0}-\frac{c \varphi_{0} \exp \left(\frac{-a^{2}}{4 \beta}\right)}{2 \sqrt{\beta}}\right)\right) .
\end{aligned}
$$

(24) shows that the expected final wealth of the PPM's can be expressed as a function of the expected value of the usual portfolio selection problem (22).

$$
\text { If we set } v_{0}=\frac{c \varphi_{0} \exp \left(\frac{-a^{2}}{4 \beta}\right)}{2 \sqrt{\beta}} \text { and } v_{0}=x_{0} \text {, }
$$

for the purpose of analysis, then, $H(T)=E(X(T))$.

Therefore, (24) becomes

$$
\begin{array}{r}
E(V(T))=\frac{1}{2} E(X(T)) . \\
\text { If } v_{0}>\frac{c \varphi_{0} \exp \left(\frac{-a^{2}}{4 \beta}\right)}{2 \sqrt{\beta}} \text { and } v_{0}=x_{0}
\end{array}
$$

then, $E(V(T))>0$.

$$
\text { If } v_{0}<\frac{c \varphi_{0} \exp \left(\frac{-a^{2}}{4 \beta}\right)}{2 \sqrt{\beta}} \text { and } v_{0}=x_{0} \text {, }
$$

then, $E(V(T))<0$.

\section{Conclusion}

This paper considered the variational form of classical portfolio strategy and expected terminal wealth for a Pension Plan Member (PPM) in a Defined Contribution (DC) Pension scheme. The present value of PPM's future contribution process was established and numerical ressults were given. The optimal portfolio processes with inter-temporal hedging terms that offset any shocks to the stochastic cash inflows were obtained. The expected value of PPM's terminal wealth and numerical results were established. 


\section{REFERENCES}

[1] L. Cao and Z. F. Guo, "Optimal Variance Swaps Investments," IAENG Journals, Vol. 41, No. 4, 2011, Article ID IJAM_41_4_07.

[2] L. Cao and Z. F. Guo, "Delta Hedging through Deltas from a Geometric Brownian Motion Process," Proceedings of International Conference on Applied Financial Economics, London, 30 June-2 July, 2011.

[3] P. E. Davis, "Portfolio Regulation of Life Insurance Companies and Pension Funds," Oxford University Press, Oxford, 2000.

http.//www.oecd.org/dataoecd/30/39/2401884.pdf.

[4] A. J. G. Cairns, D. Blake and K. Dowd, "Stochastic Lifestyling: Optimal Dynamic Asset Allocation for Defined Contribution Pension Plans," Journal of Economic Dynamics \& Control, Vol. 30, No. 5, 2006, pp. 843-877. doi:10.1016/j.jedc.2005.03.009

[5] S. Brawne, A. M. Milevsky and T. S. Salisbury, "Asset Allocation and the Liquidity Premium for Illiquid Annuities," The Journal of Risk and Insurance, Vol. 70, No. 3, 2003, pp. 509-526. doi:10.1111/1539-6975.t01-1-00062

[6] G. Deelstra, M. Grasselli and P. Koehl, "Optimal Design of the Guarantee for Defined Contribution Funds," 2002. http:/www.amazon.com/Optimal-design-guarantee-defin ed-contribution/dp/B000RQY19I.

[7] G. Deelstra, M. Grasselli and P. Koehl, "Optimal Invest- ment Strategies in the Presence of a Minimum Guarantee," Insurance: Mathematics and Economics, Vol. 33, No. 1, 2003, pp. 189-207. doi:10.1016/S0167-6687(03)00153-7

[8] G. Deelstra, M. Grasselli and P. Koehl, "Optimal Design of the Guarantee for Defined Contribution Funds," Journal of Economics Dynamics and Control, Vol. 28, No. 2, 2004, pp. 2239-226.

[9] C. R. Nwozo and C. I. Nkeki, "Optimal Investment Strategy for a Defined Contributory Pension Plan in Nigeria Using Dynamic Optimization Technique," Studies in Mathematical Sciences, Vol. 2, No. 2, 2011, pp. 43-60.

[10] C. R. Nwozo and C. I. Nkeki, "Optimal Investment and Portfolio Strategies with Minimum Guarantee and Inflation Protection for a Defined Contribution Pension Scheme," Studies in Mathematical Sciences, Vol. 2, No. 2, 2011, pp. 78-89.

[11] C. R. Nwozo and C. I. Nkeki, "Optimal Portfolio and Strategic Consumption Planning in a Life-Cycle of a Pension Plan Member in a Defined Contributory Pension Scheme," IAENG Journals, Vol. 41, No. 4, 2011, Article ID IJAM 41_4_03.

[12] A. Zhang, R. Korn and C. O. Ewald, "Optimal Management and Inflation Protection for Defined Contribution Pension Plans," Worker Paper, University of St. Andrews, 2007. http://mpra.ub.uni-muenchen.de/3300/1/MPRA_paper_33 00.pdf 